\title{
Wear damage resulting from sliding impact kinematics in pressurized high temperature water: energetical and statistical approaches
}

\author{
Anne-Laure Kaiser ${ }^{1,2}$, Sandrine Bec $^{2,4}$, Jean-Philippe Vernot ${ }^{1}$ and Cecile Langlade ${ }^{2,3}$ \\ ${ }^{1}$ FRAMATOME-ANP Technical Center, Avenue B. Marcet, Porte Magenta, 71200 Le Creusot - FRANCE \\ ${ }^{2}$ Laboratoire de Tribologie et Dynamique des Systèmes (LTDS), UMR 5513 CNRS/ECL/ENISE, Ecole \\ Centrale de Lyon, 36 Avenue Guy de Collongue, 69134 Ecully Cedex- FRANCE \\ ${ }^{3}$ Since September 2005 Laboratoire d'Etudes et de Recherches sur les Matériaux, les Procédés et les Surfaces \\ (LERMPS), Université de Technologie de Belfort Montbéliard, site de Montbéliard, 90010 Belfort - FRANCE
}

E-mail : sandrine.bec@ec-lyon.fr

\begin{abstract}
Specific wear of Rod Cluster Control Assemblies (RCCA) in Pressurized Water nuclear Reactors (PWR) results from contacts with their guides due to flow induced vibration. Particular sliding impact contact conditions and specific environment in PWR coupling temperature and solution chemistry involve original mechanisms of wear. A specific tribometer has been developed during these past years by FRAMATOME-ANP. This simulator operates in experimental conditions close to sliding impact motion of RCCA assemblies against guides and under similar environment (temperature, pressure and water chemistry). Numerous and various wear tests were realised in order to study the influence of the sliding impact parameters on wear loss and wear scars. Specific tests with continuous data acquisition were realised. Different wear scars were observed. An analogy with erosion was done. An erosion model was used to determine critical conditions concerning friction coefficient and incidence angle values leading to wear. Considering these critical conditions, it was possible to explain the observed difference of wear loss and facies.
\end{abstract}

\section{Introduction}

Some internal components of pressurised water reactor (PWR) are submitted to relative motions due to the necessary operational processes or to flow induced vibration (Ko 1997). Thus, components and associated supports, particularly Rod Cluster Control Assemblies (RCCA) are typically excited by a complex kinematics identified as sliding impacts (Zbinden and Dragon-Louiset 2000). Furthermore, typical environment in PWR, coupling high temperature $\left(320^{\circ} \mathrm{C}\right)$, high pressure $(154 \mathrm{bars})$ and solution chemistry (deaerated, low conductivity water), involves specific corrosion processes (Stellwag 1988). Extensive inspections on components of French PWR reactors have revealed, in a few cases, a significant wear on some RCCA assemblies. Worn surface observations show specifical wear scar geometry composed of several Elongated Indent Marks (EIM) and absence of plastic deformation under these surfaces.

This specific wear observed on RCCA assemblies results from original mechanisms of wear. Firstly, due to low Hertzian pressure (Ko 1987), asperities play a major role in the contact, which leads to discontinuous contact area. Secondly, Archard law isn't directly applicable because of discontinuous kinematics (sliding impacts) and environment effects. Thirdly, this specific wear depends on the temperature (Kaczorowski et al 2004) and on the water chemistry (Kaczorowski et al 2005). Moreover, wear scar geometry is atypical (Kaczorowski et al 2001). Aiming to elucidate the origin of this specific wear leads to study the influence of the mechanics, of the corrosion effects and of the combined mechanics and corrosion effects which is called tribocorrosion. In order to study these mechanisms, industrial and academic research teams have chosen to develop representative wear tests, imitating the complex kinematics, the contact geometry and the high temperature water environment (Kaczorowski et al 2001).

\footnotetext{
${ }^{4}$ Author to whom any correspondence should be addressed.
} 
Thanks to numerous and various tests realised on a specific tribometer developed by FramatomeANP, the sliding impact parameters can be detailed using statistical approaches.

This paper aims to show that the mechanical effect, even under low contact pressure conditions, plays a preponderant role in the wear of RCCA assemblies. Experimental conditions and test results are described. The reproducibility of wear tests is verified. An energy criterion is used to determine conditions leading to absence of wear, in terms of friction coefficient and impact incidence angle values.

\section{Experimental details}

\subsection{Wear simulator}

The specific tribometer developed by FRAMATOME-ANP, named AURORE, was designed to test control rod against support or fuel rod section against grid contacts under pressurized water reactor conditions (Vernot et al 2004). The original test bench, dedicated to mechanical wear processes, is an autoclave running at a temperature up to $320^{\circ} \mathrm{C}+/-5^{\circ} \mathrm{C}$ and at pressure of 154 bars. There is no circulation of the water and its chemical composition is similar to the one of primary coolant pressurised water reactor $\left(\left[\mathrm{O}_{2}\right]<0.1 \mathrm{ppm},[\mathrm{B}]: 1300 \mathrm{ppm},[\mathrm{Li}]: 2 \mathrm{ppm},\left[\mathrm{Cl}^{-}\right]<0.05 \mathrm{ppm}\right)$. Its $\mathrm{pH}$ is about 7 at $300^{\circ} \mathrm{C}$.

Figure 1a presents a schematic representation of the tribometer. The stationary sample (ring) is mounted on a flexible support of rigidity $\mathrm{K}$. Influence of the support stiffness on wear rate was evaluated by using different flexible supports with two different stiffnesses $\left(83.10^{6} \mathrm{~N} \cdot \mathrm{m}^{-1}\right.$ and $9.10^{6}$ $\mathrm{N} . \mathrm{m}^{-1}$ ). The concentric mobile sample (tube) is placed around a mast of $500 \mathrm{~mm}$ height, clamped at its bottom end and supported at the upper end. Four electro-magnets, located at $90^{\circ}$ from each other produce the controlled motion of the mobile sample.

Two sensors are used to measure normal and tangential forces and two eddy current sensors are used to obtain differential displacement of the mobile sample. Electro-magnets and dynamic data acquisition are computer controlled. Data are recorded with a high sampling rate of $32 \mathrm{kHz}$.

\subsection{Wear specimens}

Figure $1 \mathrm{~b}$ presents the samples used for this study, which are two coaxial cylinders, a ring and a tube, with a clearance of $0.5 \mathrm{~mm}$. The stationary sample is a ring of $5 \mathrm{~mm}$ height and $10.7 \mathrm{~mm}$ internal diameter. The mobile sample is a tube of $11 \mathrm{~mm}$ length, $9.7 \mathrm{~mm}$ external diameter and with a thickness of $0.95 \mathrm{~mm}$. Both samples are made of austenitic stainless steel (18 wt \% Cr, $10 \mathrm{wt} \% \mathrm{Ni}, 2$ wt $\% \mathrm{Mn}$ and $70 \mathrm{wt} \% \mathrm{Fe}$ ). The representativeness of wear tests is a very important point. That is why, specimens used in these simulators are extracted from the real components. So, the metallurgy of the materials (AISI 304L), the geometry (clearance between the ring and the tube) and the contact geometry (conformal cylinder-cylinder) are the same as in PWR.

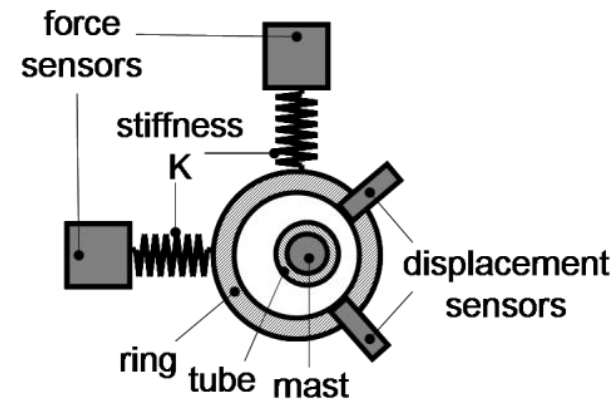

(a)

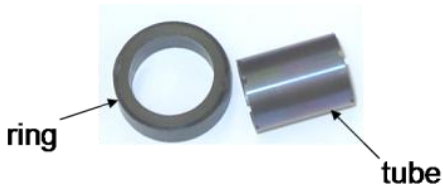

(b)

Figure 1. (a) Principle of FRAMATOME-ANP wear apparatus. Clearance between samples is intentionally exaggerated (b) Wear specimens used in the simulator. 


\subsection{Contact kinematics and sliding impact parameters}

The choice of applying sliding impact motion derivates from observations realised on scale 1 simulators or from the real components design (De Perthuis et al 1992). This motion is generated by an epicyclical electromagnetic force with a low frequency $f_{l}$ for the primary rotation around the center of the ring $O_{R}$ and a high frequency $f_{2}$ for the secondary rotation. Figure 2 presents the response of the tribometer to the driving motion. The trajectory of the tube center $O_{T}$ in the tube/ring clearance $C$ is represented. This results in superimposing orbital sliding and impact contacts. Thanks to a specific software, data processing calculates significant variables, such as normal and tangential forces $F_{N}$ and $F_{T}$, angular position $\beta$ of impacts and incidence angle $\theta$ (figure 2). Convention adopted in this paper defines that the incidence angle $\theta$ is $0^{\circ}$ for normal impact and $90^{\circ}$ for pure sliding.

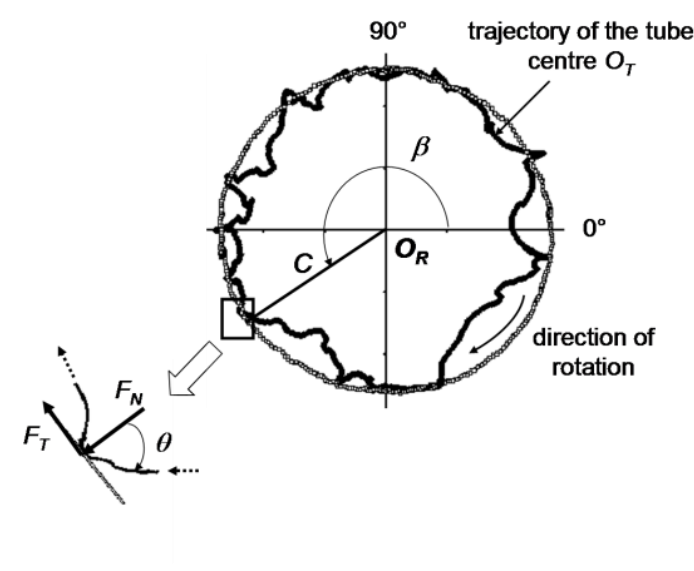

Figure 2. Motion of the tube specimen in the ring, represented by the trajectory of the tube centre $O_{T}$ in the tube-ring clearance represented by a circle of radius $C$ and centre $O_{R}$. A sliding impact is defined by its angular position $\beta$, its normal and tangential forces $F_{N}$ and $F_{T}$ and its incidence angle $\theta$.

\subsection{Experimental conditions}

Classical wear tests realised on Aurore tribometer are long duration tests $(L)$ lasting 70 hours or more. During such tests, more than 2 millions sliding impacts occur. The complete recording of the tests is not possible. Consequently, for long term tests, only a statistical recording of the data (short duration sampling every 5 hours) is done. Figure 3 shows an example of record of the normal force $F_{N}$ of a succession of sliding impacts from a 1 second record. It illustrates that the force level of each sliding impact is different with an irregular occurence. Similar observations are made with the tangential force $F_{T}$, the friction coefficient $\mu\left(F_{T} / F_{N}\right)$ and the incidence angle $\theta$. The global random behaviour mimics reality, with a high number of sliding impacts and their specific individual feature. That is why it is necessary to realise short tests $(S)$ permitting a complete recording of all parameters in order to study individually each sliding impact.

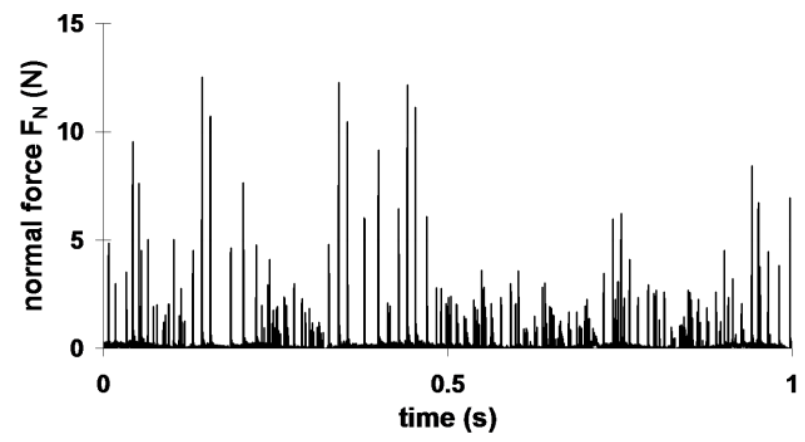

Figure 3. Normal force $F_{N}$ versus time during a 1 second record. 
The main characteristics of the wear tests presented in this paper are given in table 1. After each test, the wear specimens were analyzed. Wear volumes were determined by weighing the samples in the case of long term tests (using a balance with $0.01 \mathrm{mg}$ resolution) or estimated by 2D profilometry for short tests. Worn specimens were examined using optical microscopy and Scanning Electron Microscopy (SEM).

Table 1. Wear tests characteristics.

\begin{tabular}{ccccc}
\hline Test reference & Duration (h) & $\begin{array}{c}\text { Support Stiffness } \\
\left(\mathrm{N} . \mathrm{m}^{-1}\right)\end{array}$ & $\begin{array}{c}\text { Excitation frequencies } \mathrm{f}_{1} / \\
\mathrm{f}_{2}(\mathrm{~Hz})\end{array}$ & $\begin{array}{c}\text { Mass variation }\left(\mu \mathrm{g} \cdot \mathrm{h}^{-1}\right) \\
\text { Tube specimen }+ \\
\text { Ring specimen }\end{array}$ \\
\hline S1 & 2 & $83.10^{6}$ & $5 / 85$ & $\mathrm{NM}^{\mathrm{a}}$ \\
S2 & 2 & $83.10^{6}$ & $5 / 85$ & $\mathrm{NM}$ \\
L1 & 70 & $83.10^{6}$ & $5 / 85$ & 70 \\
L2 & 280 & $83.10^{6}$ & $5 / 85$ & 103 \\
L4 & 236 & $83.10^{6}$ & $1 / 120$ & 3 \\
L6 & 70 & $9.10^{6}$ & $10 / 200$ & 24 \\
L8 & 70 & $83.10^{6}$ & $10 / 200$ & \\
\hline
\end{tabular}

$\mathrm{NM}^{\mathrm{a}}$ : Not Measurable

\section{Results and discussion}

\subsection{Reproducibility}

Data from short tests S1 and S2 allowed us to determine the distribution of each studied parameter (see 2.3) during sliding impacts. For both tests, about 110 sliding impacts were recorded per second. Figures $4 \mathrm{a}$ and $4 \mathrm{~b}$ show the respective histograms of normal and tangential forces $F_{N}$ and $F_{T}$ and of incidence angle $\theta$ considering all the sliding impacts for the two tests. The number of sliding impacts per second, the force and the incidence angle histograms are very close for the two tests realised with the same experimental conditions, which demonstrates the very good reproducibility of the wear tests performed on Aurore tribometer.
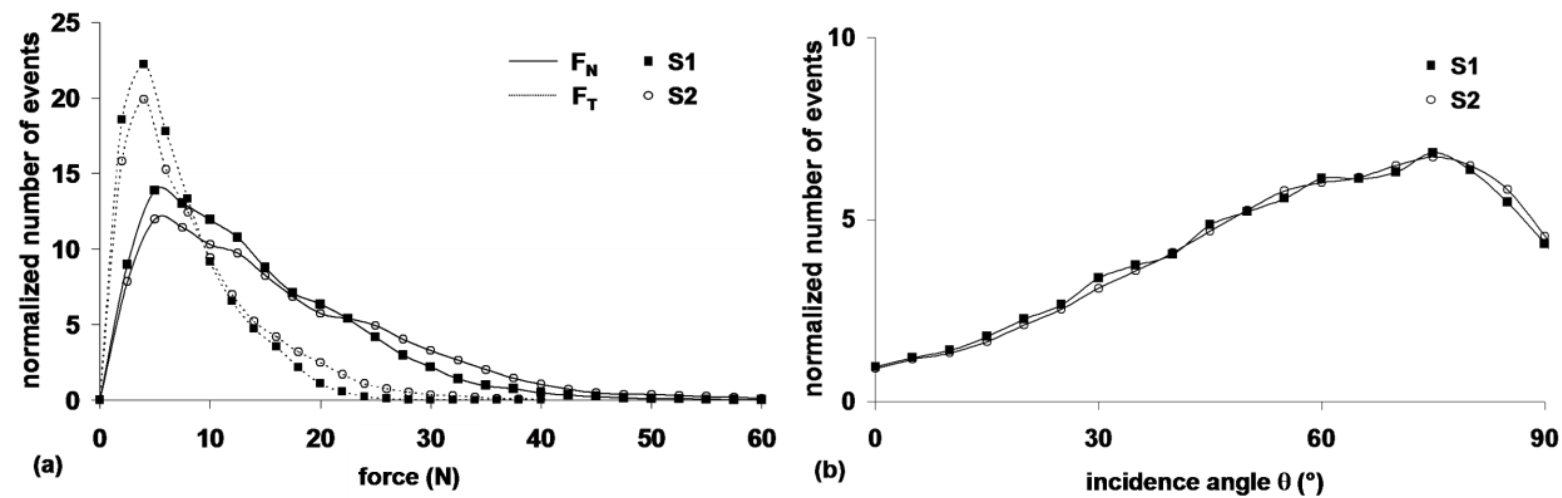

Figure 4. Comparison of (a) force histogram and (b) incidence angle histogram for wear tests S1 and S2.

\subsection{Excitation frequency and support stiffness effects}

Long term tests of different durations (between 70 and $280 \mathrm{~h}$ ), described in table 1, were realised with 3 different couples of input frequencies and 2 different support stiffnesses. For the 5 long term tests, a comparison of normal force $F_{N}$ histograms, friction coefficient $\mu$ histograms and incidence angle $\theta$ histograms (not shown here) permitted to conclude that:

- there is no evolution of sliding impact kinetics with duration as all histograms for these 3 parameters are similar for L1 and L2 tests, 
- the histograms of $\mu$ and $\theta$ depend on input frequencies (comparison between L2, L4 and L8 tests), and on support stiffnesses (comparison between L6 and L8 tests).

\subsection{Independence of friction coefficient $\mu$ and incidence angle $\theta$}

It is generally assumed that there is a link between friction coefficient $\mu$ and incidence angle $\theta$ in sliding impact conditions. Figure 5 shows the distribution of incidence angle as a function of friction coefficient for all the recorded sliding impacts of test S2. Privileged ranges of incidence angle and friction coefficient values were observed (black window on figure 5) but their large extend permits to conclude that these parameters are independent on Aurore tribometer.

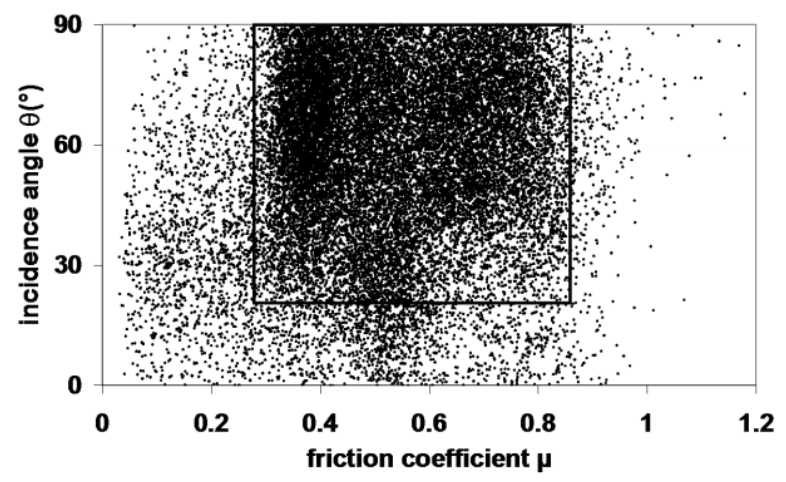

Figure 5. Incidence angle $\theta$ versus friction coefficient $\mu$ for test $\mathbf{S} 2$.

\subsection{Wear loss and wear facies}

Wear scars of specimens after long term tests are composed of many atypical Elongated Indent Marks (EIM) covering the worn surfaces. Wear loss associated to long term tests (table 1) are between 4 and $103 \mu \mathrm{g} . \mathrm{h}^{-1}$. Because of the discontinuous data recording during the long term tests, it is difficult to have a complete view of what happens during the test. So it is difficult with these tests to understand and analyse the origin of wear loss and the morphology of wear scars.

Short tests, which allow us to record all data, offer the opportunity to observe different wear scars. For both short tests and both specimens, the wear scars present two different aspects on each specimen depending on their location on the specimen. The first type of wear scars is shown on figure 6a. It is characterised by many EIM and by the presence of a friction film (interfacial film formed onto the rubbing surfaces due to friction) (Kaczorowski et al 2001). This wear scar, easily detectable on the associated topographic 2D profile is composed of valleys around $6 \mu \mathrm{m}$ deep, and peaks up to $4 \mu \mathrm{m}$ high. The second wear scar is shown on figure 6b. It is characterised by a "planished" worn surface without friction film and without significative loss of matter.

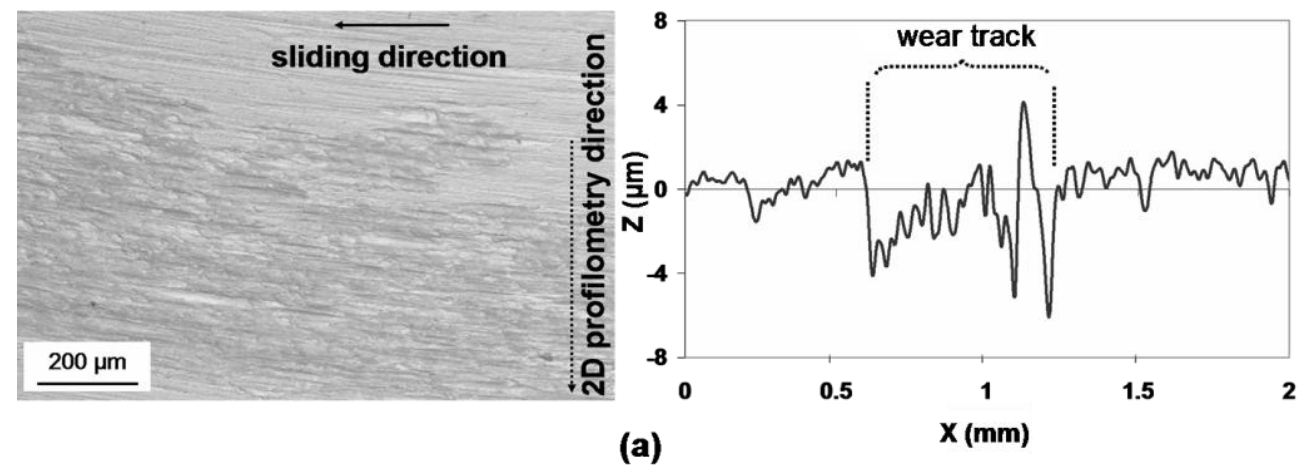




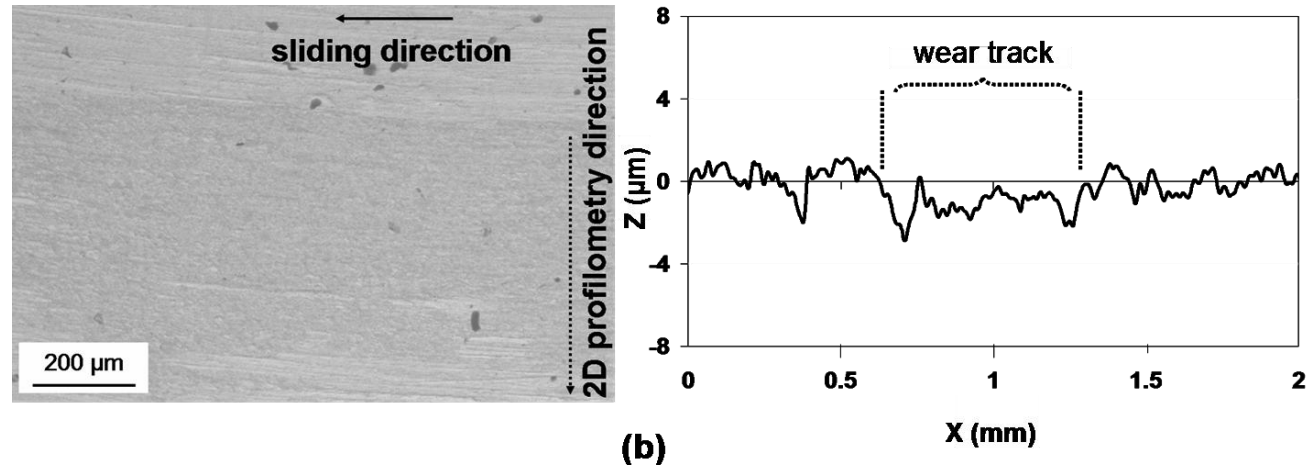

Figure 6. SEM observations and wear track profiles of worn areas of short tests (a) typical elongated indent marks, (b) typical "planished looking" area.

In order to understand the origin of worn surface differences, acquired data from 4 zones were carefully analysed on S1 and S2 specimen. Table 2 shows the different parameters relative to these 4 zones: angular position $\beta$ on the specimen, total number of sliding impacts per degree of angle $n^{*}$ obtained from the data recording and wear scar.

Table 2. Studied zones, angular position $\beta$, total number of sliding impacts per degree of angle $n^{*}$ and wear scar.

\begin{tabular}{ccccc}
\hline Zone & Angular position $\beta$ & $n^{*}$ & Wear scar & Test number \\
\hline Z1 & {$\left[135^{\circ} ;-155^{\circ}\right]$} & 1955 & EIM & S1 \\
Z2 & {$\left[-110^{\circ} ;-98^{\circ}\right]$} & 2847 & EIM & S1 \\
Z3 & {$\left[-150^{\circ} ;-135^{\circ}\right]$} & 922 & EIM & S2 \\
Z4 & {$\left[-75^{\circ} ;-60^{\circ}\right]$} & 2985 & Planished & S1 \\
\hline
\end{tabular}

Comparing $n^{*}$ values in zones $\mathrm{Z} 2$ and Z3, it can be concluded that the number of sliding impacts alone can not explain the different aspects of the wear scar. A comparison of histograms of normal force $F_{N}$ for each zone (figure 7) shows that the highest forces were measured for zone Z3, then the normal force decreases successively for zones Z4, Z2 and Z1. Thus, worn surfaces with elongated indent marks may correspond to either highest or lowest normal force $F_{N}$.

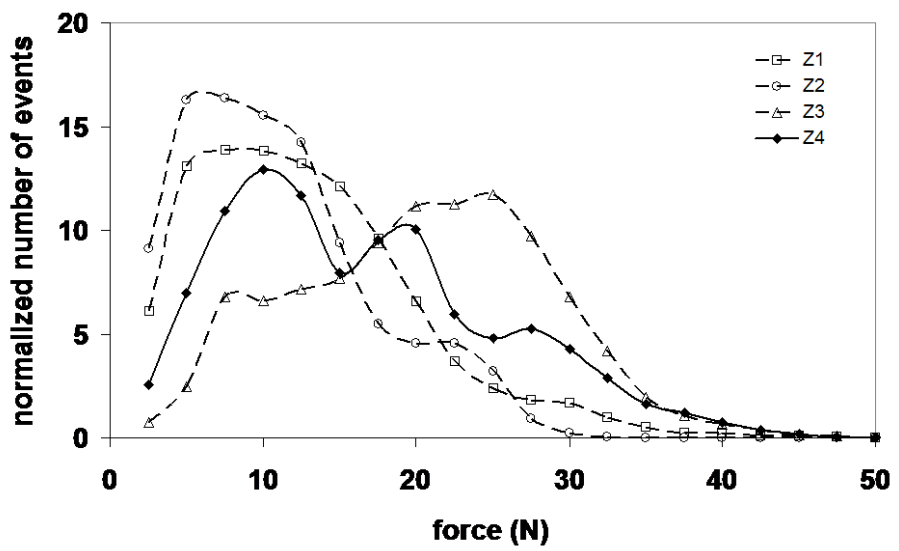

Figure 7. Comparison of a force histogram for zones Z1, Z2, Z3 and Z4.

The same comparison was realised from the histograms of friction coefficient. Zones Z1 and Z4 have similar distributions of friction coefficient, but a different aspect of wear scar.

At last, a comparison of the histograms of incidence angles showed that two different distributions of incidence angle $\theta$ can lead to the same elongated indent marks aspect of the wear scar.

So, neither number of sliding impacts nor individual sliding impact parameters, analysed separately, permit to explain the origin of the difference of worn surfaces. 


\subsection{Energy criterion}

In order to identify the critical conditions leading to the formation of elongated indent marks, a new approach of wear generated by sliding impact kinetics in specific conditions of low contact pressure is proposed.

In nuclear domain, one of the most frequently used criterion to classify wear tests is work rate (Delaune et al 2000). Work rate is a quantity deriving from normal force and tangential speed (Frick et al 1984). It is worth noting that in this criterion, the shear force is not considered, even if it has been clearly established that the shear force can be linked to wear (Ko 1985, Levy and Morry 1985). Thus, this parameter is of primary importance (Ko 1987). Nevertheless, under some simple test conditions, the work rate can be correlated either with the tangential force $F_{T}$ or with the normal force $F_{N}$ or with the total force. It should indeed be noted that in simple test conditions, a relation can be written between the shear force, the normal force, the angle of impact and the coefficient of friction (Ko 1985).

Practically, dynamic systems such as rod cluster control assemblies are subjected to complex random motion (Knudsen and Massih, 2004). The response of the tribometer reproduced this complex random motion by sliding impact kinematics. In this case, it is difficult to find wear models which take into account both the different measured wear losses and the origin of the different facies.

The works of Brach (1988) and Sundararajan (1991) in the domain of solid particles erosion show the simultaneous influence of incidence angle and friction coefficient on erosion wear of ductile materials. An analogy is proposed with wear under sliding impact conditions

The analytical model of Brach defines a normalized shear energy $T^{*}$ absorbed during contact between erosion particles and target as a function of impact angle $\alpha(\alpha=90-\theta)$, friction coefficient $\mu$, critical friction coefficient $\mu_{c}$, form factor $\lambda$ ( $\lambda=0$ for spherical particles) and restitution rate $e . \mu_{c}$ represents the maximum value of $\mu$. If $\mu<\mu_{c}$ sliding exists between the particle and the eroded material. In contrast, if $\mu=\mu_{c}$ sliding stops and the particle may roll.

The critical friction coefficient $\mu_{c}$ and the normalize shear energy $T^{*}$ are calculated with the following equations:

$$
\begin{gathered}
\mu_{C}=\frac{1}{(1+\lambda) \times(1+e) \times \tan \alpha}(1) \\
T^{*}=\frac{1}{1+\lambda} \times \frac{\mu}{\mu_{C}} \times\left(2-\frac{\mu}{\mu_{C}}\right) \times \cos ^{2} \alpha
\end{gathered}
$$

The shear energy is thus a function of friction coefficient and incidence angle. High friction coefficients produce high shear in the material. The incidence angle controls both the shear intensity of the sliding component and the hydrostatic pressure (Kermouche 2005).

In this study, an analogy between sliding impacts and hard particles erosion is proposed in order to explain the wear tests results, in terms of difference of wear scars for short tests and difference of wear loss for long term tests.

\subsection{Application to short and long term tests}

For short tests, it is necessary to determine representative ranges of incidence angle and friction coefficient for each of the previously identified zones ( $\mathrm{Z} 1$ to $\mathrm{Z} 4)$.

For the 4 zones, we can notice that the range of incidence angle distributions is large $\left[0-90^{\circ}\right]$. For each zone, a shorter range of incidence angles is defined, containing $50 \%$ of the impacts around the most frequently observed incidence angle value. The most frequent incidence angle values $\theta$ for Z1, Z2, Z3 and $\mathrm{Z4}$ are respectively $\left[55^{\circ} ; 80^{\circ}\right],\left[20^{\circ} ; 80^{\circ}\right],\left[55^{\circ} ; 80^{\circ}\right]$ and $\left[75^{\circ} ; 90^{\circ}\right]$. 
On the opposite, in each zone, the distributions of friction coefficient are Gaussian-like with small standard deviation. That is to say that friction coefficient is almost constant in each zone. Values of friction coefficient for $\mathrm{Z} 1, \mathrm{Z} 2, \mathrm{Z3}$ and $\mathrm{Z4}$ are respectively $0.45,0.75,0.85$ and 0.5 .

In figure 8 , the normalized shear energy absorbed during sliding impacts for each zone is calculated using the defined ranges of incidence angle values, friction coefficient values and making the hypothesis of spherical particles $(\lambda=0)$ and restitution rate $e=1$. Thus, equations (1) and (2) become:

$$
\begin{gathered}
\mu_{C}=\frac{1}{2 \times \tan \alpha} \\
T^{*}=\frac{\mu}{\mu_{C}} \times\left(2-\frac{\mu}{\mu_{C}}\right) \times \cos ^{2} \alpha
\end{gathered}
$$

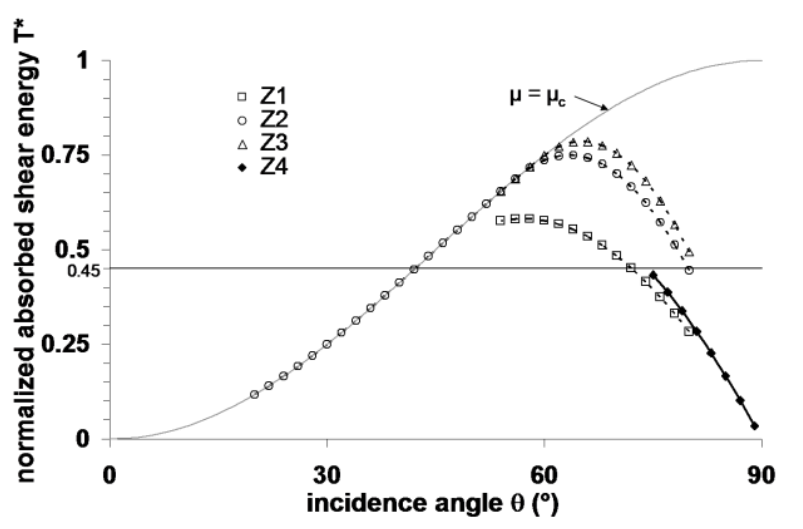

Figure 8. Correlation between friction coefficient $\mu$, incidence angle $\theta$ and absorbed shear energy $T^{*}$ as a function of friction coefficient $\mu$ and incidence angle $\theta$.

Z4 is the only zone which is characterised by a "planished looking" worn surface without significative loss of matter. On this zone, the normalized absorbed shear energy is below 0.45. Assuming the existence of a shear energy threshold below which the wear is low, this permits to conclude that this threshold is higher or equal to 0.45 . In the following, $T^{*}=0.45$ will be taken for the shear energy threshold in order to define conditions which warrant absence of significant wear i.e. non detectable loss of matter and absence of elongated indent marks.

From equations (3) and (4), it can be deducted that if the friction coefficient is lower or equal to 0.3 , the shear energy is lower than 0.45 whatever the measured incidence angle values. Furthermore, under a critical angle of $42^{\circ}$, normalized absorbed shear energy is lower than 0.45 whatever the friction coefficient. Considering the highest friction coefficient observed in short tests, with a maximum value $\mu=1$, calculation shows that for friction coefficient smaller than 1 , the shear energy is also lower than 0.45 if the incidence angle is larger than $82^{\circ}$. Thus a critical range of incidence angle can be defined. For friction coefficient between 0.3 and 1, incidence angles smaller than $42^{\circ}$ or higher than $82^{\circ}$ correspond to no-wear conditions (absence of elongated indent marks and nondetectable loss of matter).

It is also observed that few sliding impacts with high absorbed shear energy (like in Z3 for instance) create similar degradations than a lot of sliding impacts with lower absorbed shear energy (like in Z1 and Z2). So sliding impacts with friction coefficient $\mu$ lower than 0.3 , or with $\mu$ higher than 0.3 and with incidence angle $\theta$ smaller than $42^{\circ}$ or higher than $82^{\circ}$ create the lowest absorbed shear energy and are the least severe.

Thanks to this analysis, critical conditions of sliding impacts are proposed on friction coefficient $\mu$ and incidence angle $\theta$. For each long duration wear test, the percentage of sliding impacts with an incidence angle smaller than $42^{\circ}$ or higher than $82^{\circ}$, called $n_{T^{*}}$, was determined from the statistical data recordings and it was attempted to correlate the mass loss with $n_{T^{*}}$ as shown figure 9 . It should be noticed that long term tests which have the smallest number of sliding impacts $n_{T^{*}}$ are the most 
wearing tests. Nevertheless, it could be also noticed that a same percentage of sliding impacts $n_{T^{*}}$, for L1 and L2 tests, doesn't lead to the same value of mass loss. So, a 30\% uncertainty can be assumed from these results. But even with a $30 \%$ of uncertainty, the general trend is respected.

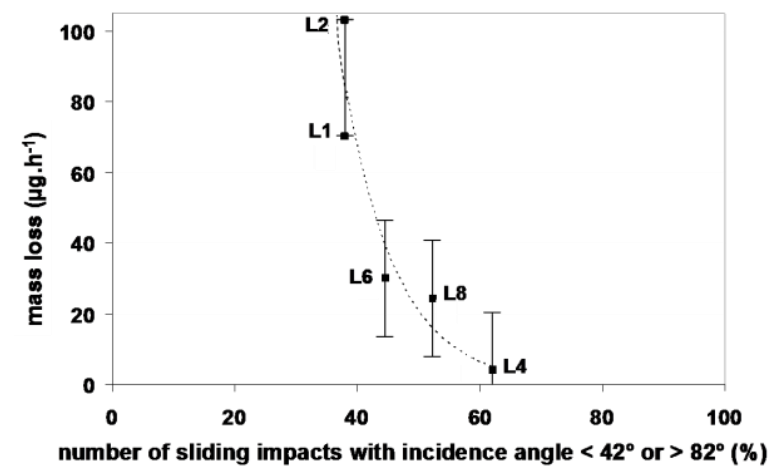

Figure 9. Evolution of mass lost per hour as a function of number of non-wearing sliding impacts.

Many reasons can be proposed to explain this high uncertainty. Firstly, long term tests are described thanks to a statistical approach and data come from short duration sampling. The quantity of recorded data is fixed by computing limits. Secondly, the model used here to determine critical conditions for sliding impacts is based on normalized shear energy and doesn't consider the impact normal force. Even if this parameter seems to be of secondary importance, it can't be neglected. Results not presented here show that with the same number of critical sliding impacts $n_{T^{*}}$, wear increases with the normal force. Introducing the normal force in the model could be an interesting improvement. Thirdly, it has been shown that test duration doesn't have any effect on sliding impact kinematics but it might have an effect in terms of stress accumulation and surface fatigue. Additional characterisation of worn surfaces has been realised. The aspect of the worn surface shown figure 10 evokes fatiguelike wear. Flaky elongated volumes, which are a mix of austenitic stainless steel and oxides are visible on the specimens. This will be further investigated in a forthcoming paper.

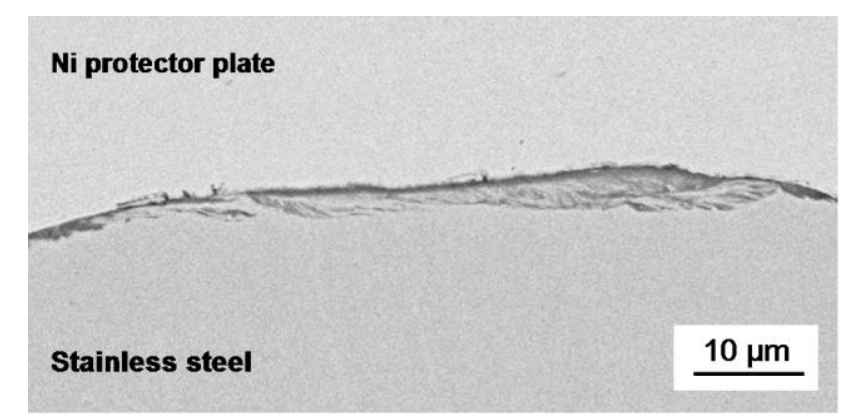

Figure 10. SEM observation of a cross section of worn surface of specimen L2.

\section{Conclusion}

Wear observed on RCCA assemblies resulting from original mechanisms of wear have been studied thanks to a specific tribometer developed by FRAMATOME-ANP. Several tests with different experimental contact conditions and duration have been realised in order to better understand the difference of wear (matter loss and wear scars).

1. The reproducibility of the wear tests performed on Aurore tribometer was demonstrated thanks to comparison of sliding impact parameter histograms issued from two tests conducted in similar experimental conditions.

2. It was noticed that there is no evolution of sliding impact kinematics with test duration.

3. Thanks to the analogy with erosion wear by hard particles, it was possible to propose a mechanical explanation to account for the difference of wear scars observed on the specimens : 
- an energy criterion was used to identify critical sliding impact conditions concerning friction coefficient $\mu$ and incidence angle $\theta$,

- a range of critical values was defined for these parameters, outside which sliding impacts do not induce significant wear,

- wear facies with elongated indent marks were associated to sliding impacts with high absorbed shear energy.

\section{References}

Brach R M 1988 Impact dynamics with applications to solid particle erosion International Journal of Impact Engineering 7/1 37-53

Delaune X, De Langre E and Phalippou C 2000 A probabilistic approach to the dynamics of wear tests Journal of Tribology 122/4 815-821

Perthuis de S, Ladouceur B, Leroux J C and Poitrenaud P 1992 Control assembly behaviour in French pressurized water reactor Kerntechnic 57/2 90-96

Kaczorowski D, Georges J M, Bec S, Vannes A B, Tonck A, Vernot J Ph 2001 Wear of a stainless steel in pressurised high temperature water Comptes rendus de l'Académie des Sciences 2/4 739-747

Kaczorowski D, Vernot J Ph and Combrade P 2005 Wear in nuclear power plants, environment effect, a tribocorrosion approach Proceedings of ICONE13 Bejing China 2005 paper number $\mathbf{5 0 5 1 5}$

Kaczorowski D, Vernot J Ph and Crenn C 2004 Wear in nuclear power plant. Proceedings of the International Symposium on Fretting Fatigue 4 Ecole Centrale Lyon France accepted to publication in Tribology International

Kermouche G 2005 Contribution à la modélisation théorique et numérique des essais d'indentation et de rayure thesis Ecole Nationale d'Ingénieurs de Saint-Etienne 2005-42

Knudsen J and Massih A R 2004 Impact oscillations and wear of loosely supported rod subject to harmonic load Journal of Sound and Vibration 278/4-5 1025-1050

Ko P L 1985 The significance of shear and normal force components on tube wear due to fretting and periodic impacting Wear 106 261-281

Ko P L 1987 Metallic wear - a review with special references to vibration-induced wear in power plant components Tribology International 20/2 66-78

Ko P L 1997 Wear of power plant components due to the impact and sliding Applied Mechanisms Reviews ASME 50/7 387-411

Levy $\mathrm{G}$ and Morri $\mathrm{J}$ Impact fretting wear in $\mathrm{CO}_{2}$-based environments Wear 106 97-138

Stellwag B 1988 The mechanism of oxide film formation on austenitic stainless steels in high temperature water Corrosion science 40/2 337-370

Sundararajan G 1991 A comprehensive model for the solid particle erosion of ductile materials Wear 149 111-127

Vernot J Ph, Kaczorowski D and Crenn C 2004 Analysis of mechanisms induced by sliding and corrosion: dedicated apparatus for PWR environments Proceedings of Eurocorr 2004 Nice France accepted to publication in Green Book "Corrosion issues in Light Water Reactors"

Zbinden M and Dragon-Louiset M 2000 Usure induite par vibrations sous écoulement : le cas des grappes de commande des réacteurs à eau pressurisée. Des études théoriques aux applications industrielles Mécanique \& Industries 1/6 581-592 